\title{
The edge complex: Implicit memory for figure assignment in shape perception
}

\author{
MARY A. PETERSON \\ University of Arizona, Tucson, Arizona \\ and \\ JAMES T. ENNS \\ University of British Columbia, Vancouver, British Columbia, Canada
}

\begin{abstract}
Viewing a stepped edge is likely to prompt the perceptual assignment of one side of the edge as figure. This study demonstrates that even a single brief glance at a novel edge gives rise to an implicit memory regarding which side was seen as figure; this edge complex enters into the figure assignment process the next time the edge is encountered, both speeding same-different judgments when the figural side is repeated and slowing these judgments when the new figural side is identical to the former ground side (Experiments 1A and 1B). These results were obtained even when the facing direction of the repeated edge was mirror reversed (Experiment 2). This study shows that implicit measures can reveal the effects of past experience on figure assignment, following a single prior exposure to a novel shape, and supports a competitive model of figure assignment in which past experience serves as one of many figural cues.
\end{abstract}

The assignment of figure and ground status to portions of an image is among the most seemingly effortless aspects of visual perception. Yet the consequences of this assignment are profound. A region (or a portion of a region) perceived as figure has a definite shape, meaning that its bounding edges are assigned as belonging to it. When figure assignment is determined, the polarity of the segments of the edge (e.g., which segments are convex or concave) is defined with respect to the figure. In contrast, a region (or a portion of a region) that is perceived as ground does not have a definite edge where it adjoins the figure, since the ground is treated as only the visible portion of a larger shape that extends behind the figure that partly occludes it. ${ }^{1}$

Early studies of figure assignment were based on the direct reports of observers viewing images consisting of black and white regions (e.g., Kanizsa \& Gerbino, 1976; Rubin, 1915/1958). These studies identified factorshenceforth called classic configural cues - that influence figure-ground assignment. For instance, image regions that are relatively smaller, more enclosed, and more con-

This research was conducted while M.A.P. was supported by the National Science Foundation (BNS 9906063 and BCS 0418179) and J.T.E. was supported by the Natural Sciences and Engineering Research Council of Canada. This project could not have been done without the dedication and hard work of Stephanie Woods Beverage and Andrea Soulé. Thanks also to Diana Olivas for her help with Experiments 1 and 2 and to Stephen Palmer, Robert Rauschenberger, Emily Skow-Grant, and two anonymous reviewers for their comments on a previous draft. Correspondence concerning this article should be addressed to M. A. Peterson, Department of Psychology, University of Arizona, 1503 E. University Blvd., Tucson, AZ 85721 (e-mail: mapeters@u.arizona.edu). vex are more likely to be seen as figure and, therefore, in ownership of the edge between regions (for reviews, see Hochberg, 1971; Palmer, 1999; Peterson, 2000; Pomerantz \& Kubovy, 1986).

A traditional assumption has been that the classic configural cues were used to determine which region was figure before memory traces of previously seen shapes or objects were accessed. On this view, figure assignment was immune to the effects of past experience, which had an influence only after figure assignment was complete. Before the early 1990s, very few experiments were even designed to investigate whether or not past experience could affect figure assignment, and those that did produced contradictory results (for a review, see Peterson, 1999). More recently, Peterson and her colleagues (e.g., Peterson \& Gibson, 1994) have shown that past experience with particular shapes and objects does exert an influence on figure assignment. Those conclusions have not gone unchallenged, however. In what follows, we will briefly discuss the evidence pro and con the conclusion that past experience affects figure assignment. We then will present three experiments designed to resolve the debate.

\section{Evidence That Past Experience Affects Figure Assignment}

Peterson and her colleagues (see Peterson \& SkowGrant, 2003, for a review) used stimuli in which an edge bordering two regions depicted a portion of a well-known shape (e.g., a portion of a sea horse or a woman) along one side, but not along the other. They presented such stimuli to observers in two orientations: upright, in which the known object was portrayed in the orientation in 
which it is typically seen, and inverted, in which the known object was portrayed upside down. In a number of experiments, they consistently found that the observers were more likely to perceive the figure on the side of the edge where the known object was depicted when the stimuli were upright, rather than inverted. The change in orientation altered the familiarity of the view of the object but did not change the classic configural cues present in the display. Accordingly, Peterson and colleagues took the orientation-dependent change in perception as evidence that figure assignment is affected by past experience with known shapes.

Peterson and Gibson (1994) found that past experience does not determine figure assignment exclusively but that its influence is similar to the classic configural cues in contributing evidence in favor of a particular figure assignment. They proposed that prior to figure assignment, both sides of an edge are evaluated for both the classic configural cues and for matches to memory traces of previously seen shapes or objects. These memory traces, called edge complexes, are traces of configured portions of an edge (larger than a single feature or part and smaller than the whole object or edge) that indicate where a shaped entity was previously located with respect to the edge (Peterson \& Lampignano, 2003). To illustrate how past experience can interact with classic configural cues, Peterson, de Gelder, Rapcsak, Gerhardstein, and Bachoud-Lévi (2000) proposed the competitive model of figure assignment shown in Figure 1. In this model, configural cues (including past experience) on the same side of an edge cooperate to boost the likelihood that the figure will be seen to lie on that side of the edge. Configural cues on opposite sides of an edge compete. It is assumed that the cross-edge competition takes time. One outcome of the cross-edge competition

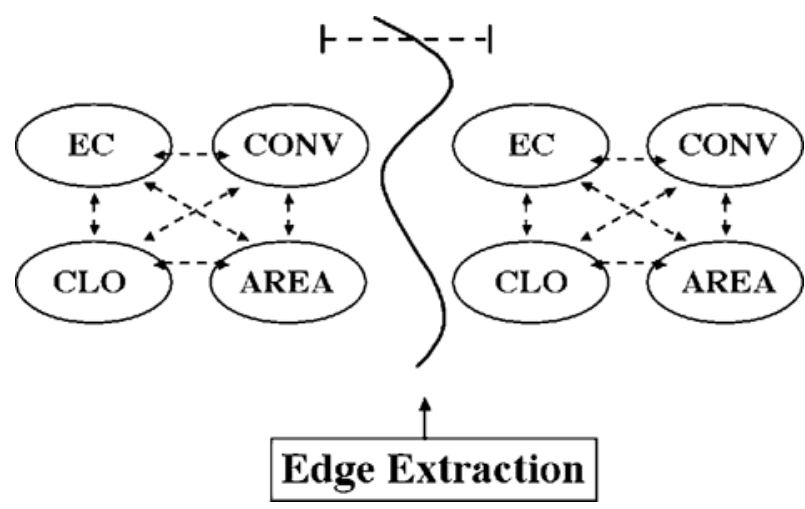

Figure 1. The competitive model proposed by Peterson, de Gelder, Rapcsak, Gerhardstein, and Bachoud-Lévi (2000). Cues on the same side of an edge cooperate (double-headed arrows); cues on opposite sides compete (double-headed sideways T). As a result of these interactions, a shape is perceived on one side of an edge (the figure side). The other, more weakly cued side is perceived to be shapeless near the shared edge. $\mathrm{EC}$, edge complex; CLO, closure; CONV, convexity. is that the shaped figure is perceived to lie on the more strongly cued side of the edge; another outcome is that the more weakly cued side of the edge appears shapeless.

In contrast to this position, Driver and Baylis (1995, 1996; Baylis \& Cale, 2001; Baylis \& Driver, 1995) presented results that they took as evidence against the position that past experience affects figure assignment. In what follows, we will discuss the contradictory evidence, the assumptions on which it was based, and how it was obtained. We then will report a series of experiments adapting Driver and Baylis's (1996) paradigm, to attempt to resolve these apparently contradictory results.

\section{Evidence Taken to Indicate That the Ground Side of an Edge Is Not Processed}

Driver and Baylis (1996) first presented observers briefly with a rectangular display in which two adjacent regions of different color shared a stepped border (see Figure 2A). One of these regions was always smaller and brighter $^{2}$ than the other, with the intent that it would be seen as the shaped figure and the adjacent region would be seen as a shapeless ground. The explicit task given observers was to remember the shape of the contour (edge) separating the two regions. This study display was exposed briefly, and, following a short delay, a pair of probe shapes appeared, one located above the other (see Figures $2 \mathrm{~B}$ and $2 \mathrm{C}$ ). The probes were expected to be seen as figure against the background on which they were displayed because they were small, enclosed regions. Both of the probe shapes had stepped borders, but only one had the same stepped border as that in the study display. Observers made a speeded response to indicate which of the probe shapes had the same stepped border as the study display.

The critical manipulation included two types of probe displays: In figure probes (Figure 2B), the shape lay on the same side of the stepped border as it had in the study display; in ground probes (Figure 2C), the shape lay on the opposite side (i.e., on the same side as the shapeless ground in the study display). ${ }^{3}$ The two probe shapes shown on a given trial were of the same type; both were either figure probes or ground probes. The results showed that the probe contour matching task could be accomplished more quickly and accurately for figure probes than for ground probes. That is, the observers were unable to remember the contour in isolation. Instead, they remembered it as a border of the smaller, brighter region in the study display (i.e., the figure). This finding spoke to the issue of the automatic nature of figure assignment, since the task did not formally require that a figure be assigned in the study display.

What is critical for the present discussion is that Driver and Baylis $(1995,1996)$ also took their results as evidence that the ground side of an edge is not evaluated for past experience prior to figure assignment. Had such an evaluation taken place, they reasoned, the observers would have been able to respond as rapidly to ground probes as they did to figure probes. This prediction can be 


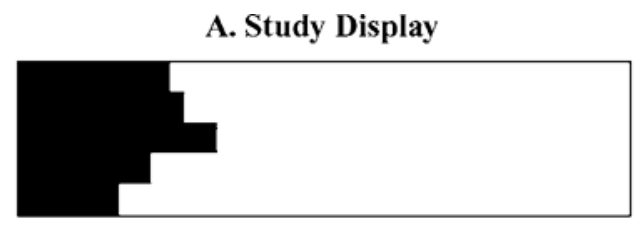

\section{B. Figure Probes}
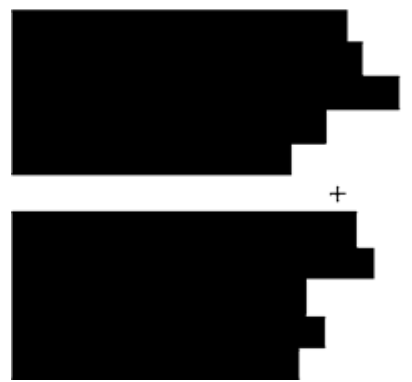

\section{Ground Probes}
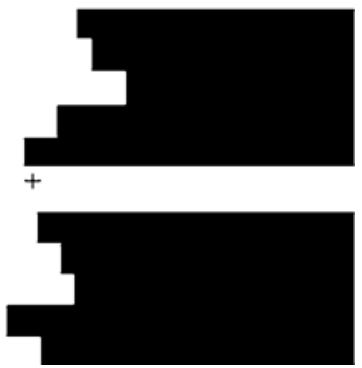

Figure 2. Illustration of the study and probe displays used by Driver and Baylis (1996). (A) In the study display, two adjacent regions shared a stepped edge; one region was smaller in area and of higher contrast (i.e., yellow in Driver \& Baylis's study, shown in black here) than the other region, which was red (shown in white here). The larger area, shown in white here, is outlined in black because the scene background in the illustration is white. Driver and Baylis's displays did not contain outlines. ( $B$ and $C$ ) Figure and ground probes, respectively. Classic configural cues advocate that the figure lies on the same side of the stepped edge as in the study display in figure probes and on the opposite side in ground probes. For both figure and ground probes shown here, the stepped edge in the top probe matches the stepped edge in the study display. In the experiment, the matching edge was in the top probe and the bottom probe equally often.

made only on the assumption that prior to the determination of figure and ground in the study display, equivalent representations were formed for the potential shape of both the figure and the ground. However, we note that this is not a prediction that follows from Peterson's competitive model of figure assignment. ${ }^{4}$ Given that the ground side of the study display was determined to be shapeless near the stepped edge, the Peterson model assumes that no memory of the shape of the ground was established. Indeed, as we will discuss next, Driver and Baylis's results would be expected if, prior to figure assignment, both sides of the edge of the probe display were evaluated for configural cues, including past experience.

\section{Competitive Model Predictions for the Driver and Baylis (1996) Task}

Consider the cross-edge competition that is proposed to occur when the study display is presented. The stepped edge is always novel, and so past experience cannot serve as a cue on either side of the edge. The balance of configural cues is such that the small bright region wins the competition as figure, whereas the larger, dimmer region is evaluated to be ground at the edge it shares with the figure. As a result of the cross-edge competition, a memory trace is established, indicating where the figure lies with respect to the configuration of parts along the stepped edge shared by the two adjoining regions (Peterson \&
Lampignano, 2003). ${ }^{5}$ The next time this edge is encountered (i.e., on the probe displays), its memory trace, called an edge complex, will be available to serve as a cue based on recent past experience.

Indeed, Driver and Baylis's (1996) data can be predicted if one posits the operation of an edge complex that was established during exposure to the study display and was reactivated when the contour of the study figure was repeated on probe trials, serving as a cue with which to assign the figure on the same side of the edge. For figure probes, this experience-based cue cooperated with the classic configural cues of small area and enclosure and with the observers' task set to judge the shape similarity of the two small, enclosed probes. In contrast, for ground probes, this same past experience cue was in conflict with both the classic configural cues and the observers' task set. Past experience cannot be expected to dominate the figure assignment outcome for ground probes, because more cues specify that the figure lies on the opposite side of the repeated edge. However, the presence of competition from past experience might increase the time taken to resolve the cross-edge competition and, therefore, might be reflected in longer response times (RTs) for ground probes than for figure probes (cf. Peterson \& Lampignano, 2003).

This discussion shows that Driver and Baylis's (1996) task cannot be used to adjudicate between their account, 
in which the side ultimately seen as ground is never processed for past experience, and an account in which both sides of an edge are processed for past experience before figure assignment, because RTs are expected to be longer for ground probes than for figure probes on both accounts. The question can properly be addressed only by comparing RTs to ground probes in which an edge is repeated from the study display with RTs to control ground probes with novel edges. A competitive model in which both sides of an edge are assessed for past experience prior to figure assignment would predict longer RTs to ground probes with repeated edges than to control ground probes with novel edges. In contrast, Driver and Baylis's account would predict that RTs to the two types of ground probes would be equivalent. Unfortunately, the comparison between novel control ground probes and ground probes with an edge repeated from the prime display is not possible using Driver and Baylis's method, because in their explicit memory task, one of the probes must share a border with the figure in the previous study display.

Since Driver and Baylis's (1996) study, three studies have addressed similar issues, using control probes. Two of the studies (Peterson \& Lampignano, 2003; Treisman \& DeSchepper, 1996, Experiment 6) reported results consistent with the predictions of the competitive model; one (Baylis \& Cale, 2001) reported results favoring the predictions we derived from Driver and Baylis. Notably, different methods were used in these studies. We will discuss those experiments next and then will present the design used in the present experiments, which adapted features of each of the methods used in these studies and of the original method used by Driver and Baylis (1996).

\section{Previous Experiments Using Control Probes}

In the studies of Treisman and DeSchepper (1996, Experiment 6) and Peterson and Lampignano (2003), prime and probe trials were paired. On the first trial of the pair, the prime trial, observers viewed a standard novel shape, shown above fixation, and reported whether or not it was the same as a comparison shape shown below fixation. Both the standard and the comparison shapes were small, black, closed silhouettes with three straight edges and one articulated curved edge. The curved edge lay on either the left or the right side of the shape; hence, the black shapes could be described as facing in the direction of their curved edge, to the right or the left. On the second trial of each pair, the probe trial, the observers saw a white standard shape above fixation and reported whether or not it was the same as a white comparison shape shown below fixation. The white shapes shown on the probe trials always faced in the opposite direction from that in which the black shapes on the prime trials had faced. Half of the time, the curved edge of the white standard shape was repeated from the standard black shape shown on the prime trial (experimental ground probes); the other half of the time, the curved edge of the standard white shape was novel (control ground probes). ${ }^{6}$ In both studies, same-different RTs were longer on ex- perimental than on control ground probe trials, as would be predicted if an edge complex established on the prime trial had been accessed in the course of figure assignment in the probe trial. (See Peterson and Lampignano, 2003 , for more on the distinction between this interpretation and Treisman and DeSchepper's, 1996.)

One previous study employing control trials failed to show different RTs for experimental and control ground probes (Baylis \& Cale, 2001). Several procedural differences may have contributed to these different results, including the fact that (1) the shapes were horizontally, rather than vertically, oriented, as they had been in previous studies, (2) the task involved judging the symmetry of a single shape, such that a response could be generated using either the figure or the ground side of the edge, and (3) both figure and ground probes were included, instead of only ground probes. With this design, Baylis and Cale found that RTs were shorter to experimental than to control figure probes but that responses to experimental and control ground probes did not differ. The absence of a difference in responses to experimental and control ground probes is consistent with Driver and Baylis's (1996) claim that the ground side of an edge is not processed for past experience prior to figure assignment. But this null effect stands in contrast to the differences between responses to experimental and control ground probes obtained by Treisman and DeSchepper (1996, Experiment 6) and by Peterson and Lampignano (2003).

In attempting to explain why their results differed, Baylis and Cale (2001) pointed out that Treisman and DeSchepper's (1996) participants may have actively ignored the ground region of their prime displays in order to perform the discrimination task on the black regions. In this account, slower responses to experimental than to control ground probes reflected the need to attend to stimuli that had previously been ignored, ${ }^{7}$ an interpretation that is orthogonal to the questions regarding figure and ground assignment.

\section{Precis}

In the present study, we investigated directly whether or not differences between responses to experimental and control ground probes could be obtained in a design that combined features of the designs used by Treisman and DeSchepper (1996) and by Driver and Baylis (1996). Our stimuli were modeled after those used by Driver and Baylis (1996). Addressing the concern raised by Baylis and Cale (2001), we did not ask the observers to respond to the prime display; we asked them simply to look at it. Our observers' task was to decide whether two probe shapes shown one above the other on a subsequent frame were the same as or different from each other. No explicit reference back to the prime was necessary.

The design included both figure and ground probes, following Driver and Baylis (1996) and Baylis and Cale (2001). On any account, one would expect observers to respond faster to experimental than to control figure 
probes (e.g., Beller, 1971; Jacoby, 1983; Rosch, 1975; Scarborough, Cortese, \& Scarborough, 1977; Sekuler \& Palmer, 1992). This standard priming effect need not reflect processes involved in figure assignment. The critical prediction for distinguishing the account of Driver and Baylis (1996) from that of Peterson and her colleagues concerns the ground probes. If Baylis and Cale are correct - namely, that Treisman and DeSchepper obtained longer RTs for experimental than for control ground probes because their observers had to ignore the ground in order to respond to the prime-no differences in RTs to experimental and control ground probes should be obtained in the present study, because the observers do not have to make any judgments regarding the prime shape. Alternatively, if mere exposure to a novel figure on a prime trial is sufficient to establish an edge complex that can exert an influence on figure assignment the next time the novel edge is encountered, RTs to experimental ground probes should be longer than those to control ground probes.

We report three priming experiments of this type. In Experiment 1A, we used a stimulus sequence very similar to that employed by Driver and Baylis (1996). In Experiment $1 \mathrm{~B}$, we shortened the exposure duration of the prime and used a backward mask to introduce noise between the prime and the probe and to reduce, as much as possible, the effects of deliberate visual inspection of the prime. The results from both experiments were remarkably similar. First, we found that RTs to experimental figure probes were shorter than RTs to control figure probes. This confirms the fact that repeated presentations of the same figure results in a processing benefit. Second, contrary to Driver and Baylis's (1996) conclusion with regard to ground regions, we found equally strong evidence that past experience was accessed for the ground of the probe displays. In particular, RTs were longer and errors were greater for experimental ground than for control ground probes. This supports the idea that an edge complex established on the basis of a single exposure to a prime slows responses when it is inconsistent with the ensemble of figural cues in the probe and may speed responses when it is consistent. In Experiment 2, we introduced facing direction as a variable and found that the past experience cue operates over right-left reversals of the facing direction of the edge.

\section{EXPERIMENT 1}

\section{Method}

Participants. The participants were members of the University of Arizona community. Of the 31 participants who took part in Experiment $1 \mathrm{~A}, 8$ were excluded because they made errors on more than $15 \%$ of the trials (a criterion established following Driver \& Baylis, 1996). ${ }^{8}$ Of the 23 remaining, 13 were female, and 22 were right-handed. Of the 25 participants in Experiment 1B, 1 was eliminated due to an error rate greater than $15 \%$. Of the remaining 24 , 8 were female, and 23 were right-handed. Most of the participants were undergraduates who took part in the experiment for class credit ( $N=17$ and 20 in Experiments $1 \mathrm{~A}$ and $1 \mathrm{~B}$, respectively). One participant in Experiment 1B was a naive research assistant; the remaining participants were paid $\$ 15$ for their time. All had vision that was normal or corrected to normal.

Stimuli and Materials. Sample stimuli are shown in Figure 3. Primes were created off line, using a program that generated a stepped edge within a horizontally elongated rectangle, $22 \mathrm{~cm}$ wide $\times 5.5 \mathrm{~cm}$ high (see Figure $3 \mathrm{~A}$ ). The stepped edge was created via a sequence of random left, right, and downward steps from a starting position located $5.5 \mathrm{~cm}$ to the right of the top left corner of the rectangle. A different random number seed guaranteed that each of 1,024 primes was unique. There were five vertical steps in the stepped edge of each prime stimulus, each approximately $1.1-1.15 \mathrm{~cm}$ in size; two consecutive steps could not be taken in the downward direction. Steps in the horizontal direction varied in size from 0.46 to $0.92 \mathrm{~cm}$. No more than four consecutive steps could be taken in the same horizontal direction, with the added constraint that the stepped edge did not extend more than $1.8 \mathrm{~cm}$ on either side of the starting position. The smaller area to the left of the stepped edge was colored bright yellow (luminance $=17.0 \mathrm{fL}$ ); the larger area to the right was colored dark red (luminance $=1.3 \mathrm{fL}$ ). The yellow re-

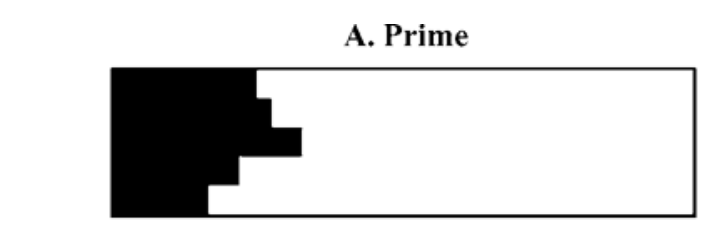

Figure Probes

B. Experimental Ground Probes

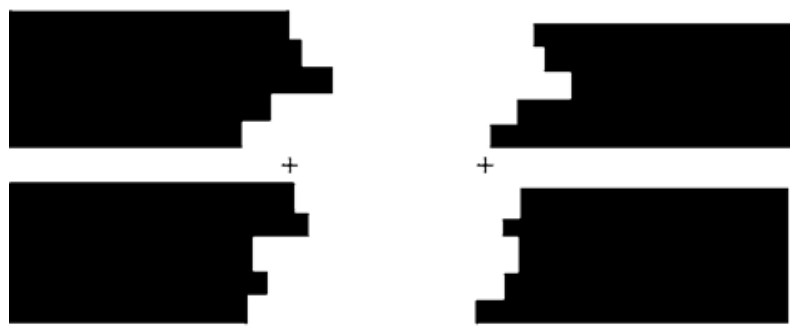

C. Control
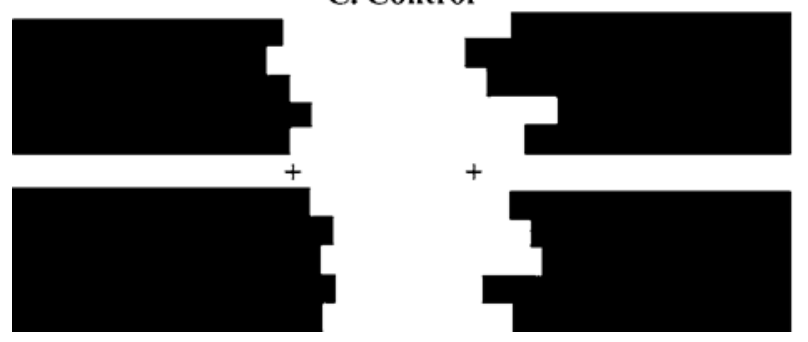

Figure 3. Illustration of the prime $(A)$ and probe $(B$ and $C)$ displays used in the present study. In panel $A$, the smaller region in the prime is black, and the larger area is white; in the experiments, these colors were bright yellow and dark red, respectively. The larger area, shown in white here, is outlined in black because the background in the illustration is white. Our displays did not contain outlines. (B and C) Experimental probes and control probes, respectively. Left: figure probes. Right: ground probes. On each trial, two probes were shown, one above and one below fixation. In this illustration, all of the probe trials are different trials, and for experimental probes, the stepped edge in the top probe matches the stepped edge in the prime. In the experiment, probe edges were never repeated; half of the probe trials were same trials; and on different trials, the stepped edge in the bottom probe matched the stepped edge in the prime half the time. 
gion accounted for approximately one fourth the total area of the rectangle. The rules for generating these stimuli were based on Driver and Baylis (1996).

After they had been created, half of the primes were mirror reversed so that the small yellow region was located on the right, creating an equal number of primes with figures on the right and the left. Half of each of these subsets of primes was paired with experimental probes (see below); the other half was paired with control probes.

The probes were created off line, using the same program as that used to make the primes. The probes averaged $11 \mathrm{~cm}$ wide and were $5.5 \mathrm{~cm}$ high. Our intention, following Driver and Baylis (1996), was to present probes that were midway in size between the figure and the ground regions of the prime. Of the probes, half $(n=512)$ were experimental probes, in that each had the same stepped edge as the prime with which it was paired. Half of the experimental probes were figure probes, in that the probe shape lay on the same side, relative to the stepped edge, as the small bright figure had in the prime (experimental figure probes). The rest of the experimental probes were experimental ground probes, in that the probe shape lay on the opposite side of the stepped edge (see Figure 3).

An additional 1,024 novel stepped borders were created. Of these, 256 were paired with experimental probes on the experimental different trials (half of these were paired with experimental figure probes and half with experimental ground probes). The remaining 768 novel stepped edges were used for control trials. Of these, half were used to create control figure probes, where the probe shape lay on the same side relative to the stepped edge as the figure had in the paired prime, and half were used to create control ground probes, where the probe shape lay on the opposite side of the stepped edge as the figure had in the paired prime; 512 of these control probes were shown on different trials (balanced across control figure and control ground probes), and 256 were shown on same trials (again balanced across control figure and control ground probes). Thus, there were an equal number of same and different probe trials.

A horizontally elongated rectangular red and yellow checkerboard mask was used in Experiment 1B. The dimensions of the mask were the same as those of the prime. The checkerboard squares were $7-8 \mathrm{~mm}$ on a side, subtending approximately $0.5^{\circ}$.

The large monitor screen background $(33 \mathrm{~cm}$ wide $\times 25 \mathrm{~cm}$ high) on which the displays were presented was intermediate gray $(7.9 \mathrm{fL})$. On half of the probe trials within each condition, the probes were white (luminance $=18.6 \mathrm{fL}$ ); on the other half of the trials, the probes were black (luminance $=0.24 \mathrm{fL}$ ). These achromatic colors were chosen for the probes so that half would be similar to the bright figure and the other half would be similar to the dark ground of the prime (although Driver \& Baylis, 1996, reported that their results did not depend on the contrast of the probe).

A chinrest was used to maintain a constant viewing distance from the monitor. A button box with two horizontally arranged buttons was used to record the latency and accuracy of the participants' same/different responses. The DMDX application was used to run the program and to record the data (Forster \& Forster, 2003).

Procedure. Instructions were presented on the computer monitor; the participants read them at their own pace. The participants were informed that they would see two displays on each trial in the experiment. The first, red and yellow, display would be shown briefly. They were told that they did not have to respond to that display. The second display would contain two shapes of the same color, either black or white, shown one above the other on either the left or the right side of the screen. Their task was to decide whether these two shapes were the same as, or different from, each other. They were instructed to respond as rapidly as possible without making mistakes. The participants were instructed to keep their eyes at the center of the screen during the sequence of events on each trial and that the black and white shapes would be readily visible without moving their eyes.

A block of 128 practice trials followed the instructions. The experimenter stayed in the room during the practice block, to ensure that the participant understood the task and to answer any questions. (Performance on the practice block was not analyzed.) Following the practice block, there were seven experimental blocks. Each block included 128 trials - 4 trials of each of 32 types of probe trials created by the factorial combination of condition (experimental/ control), probe type (figure/ground), probe color (black/white), side of prime figure (left/right), and response (same/different); each trial type was represented twice in 64-trial subblocks. The order of presentation of trials was randomized within each subblock, between subblocks, and among the seven experimental blocks. ${ }^{9}$

On each trial in Experiment 1A, a prime display was exposed for $180 \mathrm{msec}$, centered on the screen. Next, a blank gray screen was shown for $500 \mathrm{msec}$ and was followed by a probe display. These display parameters closely matched those used by Driver and Baylis (1996). On each trial in Experiment 1B, the prime display was exposed for $128 \mathrm{msec}$ and was followed immediately by a 128 -msec mask, which was followed immediately by the probe display. The probe displays contained a fixation cross that was centered on the screen and the two probe shapes; the lower edge of one probe shape was $8 \mathrm{~mm}$ above the fixation cross, and the upper edge of the other probe was $8 \mathrm{~mm}$ below it. The probes were presented so that the starting position for their stepped edges aligned with the fixation cross. Consequently, the stepped edge location was different in the prime and the probe displays (as it was in Driver \& Baylis, 1996). The fixation point and the probe displays remained on the screen until the participant responded or for 1,500 msec in Experiment 1A and 2,000 msec in Experiment 1B. ${ }^{10}$ If no response was made within this interval, the trial timed out, and the response was scored as an error. Trials on which errors were made were not repeated. A blank gray screen was exposed for $500 \mathrm{msec}$ between the offset of the probe display and the onset of the prime display for the next trial.

Following each trial block, the participants were given feedback. If their error rate was more than $15 \%$ in the preceding block, the instruction "Please try to be more accurate" was displayed. If their error rate was less than $5 \%$ in the preceding block, the instruction "Please try to respond more quickly" was displayed. The participants pressed the foot pedal to remove the feedback display. Next, the instruction "Please take a break" was displayed until they pressed the foot pedal to continue to the next block of trials. If their error rate was greater than $5 \%$ but less than $15 \%$, only the instruction "Please take a break" was displayed until the participant pressed the foot pedal to continue to the next block of trials. This feedback was modeled after that used by Driver and Baylis (1996).

The participants viewed the displays from a distance of $96 \mathrm{~cm}$. At this distance, the prime display was $12.9^{\circ}$ wide $\times 3.3^{\circ}$ high, the average width of the small figural region within the prime was $3.3^{\circ}$, and the average width of the visible portion of the larger ground region was $9.6^{\circ}$. The probes were $3.3^{\circ}$ high, with an average width of $6.5^{\circ}$. Each vertical step in the stepped edge was approximately $0.67^{\circ}$, each horizontal step varied from $0.27^{\circ}$ to $0.55^{\circ}$, and horizontal excursions were limited to lie within $1.1^{\circ}$ of the starting position. The distance between the fixation cross and the nearest edge of a probe was approximately $0.5^{\circ}$.

Half of the participants used the left button on the button box to make a same judgment and the right button to make a different judgment. This response key mapping was reversed for the other half of the participants. There were a total of 896 trials in the experimental blocks, 28 trials for each of the 32 possible prime-probe couplings in each block. Hence, for each participant, RTs were recorded on 224 trials for each of the four main probe types (i.e., experimental figure probes, control figure probes, experimental ground probes, and control ground probes). Participation took 1.25-1.5 h.

\section{Results}

Before the RT data were analyzed, trials on which errors were made were excluded. For each condition, any RTs more than two standard deviations from each participant's 
mean were eliminated recursively. This trimming procedure resulted in the elimination of $10.4 \%$ and $12.6 \%$ of the responses in Experiments $1 \mathrm{~A}$ and $1 \mathrm{~B}$, respectively. ${ }^{11}$

Mean correct RTs and mean proportions of errors for the four different types of probes are shown in Figure 4. These data were subjected to analyses of variance (ANOVAs) that were conducted to examine the influences of five factors: probe type (figure or ground), probe condition (experimental or control), probe color (black or white), response (same or different), and side of figure in the prime display (left or right). In both Experiments $1 \mathrm{~A}$ and $1 \mathrm{~B}$, the interaction between the primary factors of probe type and probe condition was significant for both RT $\left[F(1,22)=5.43, M S_{\mathrm{e}}=1,794, p<.03\right.$, for Experiment $1 \mathrm{~A}$, and $F(1,23)=9.28, M S_{\mathrm{e}}=3,250, p<.01$, for Experiment 1B] and errors $\left[F(1,22)=17.90, M S_{\mathrm{e}}=\right.$ $0.002, p<.001$, for Experiment $1 \mathrm{~A}$, and $F(1,23)=13.87$, $M S_{\mathrm{e}}=0.005, p<.01$, for Experiment 1B].

In both experiments, there were four main findings involving the theoretically critical probe type $\times$ probe condition interactions that were examined more closely with Fisher least significant difference (LSD) tests.

1. RTs to experimental figure probes were shorter than RTs to control figure probes $[F(1,22)=5.55, p<.03$, in Experiment $1 \mathrm{~A}$, and $F(1,23)=5.07, p<.04$ in Experiment $1 \mathrm{~B}]$. The finding that RTs were shorter for experimental figure probes than for control figure probes may simply reflect repetition priming - the figures in the prime and the probe displays were almost identical (the probe figures were elongated horizontally, relative to the prime figures).

2. In both experiments, errors were greater for experimental ground probes than for control ground probes $[F(1,22)=27.93, p<.001$, in Experiment $1 \mathrm{~A}$, and $F(1,23)=19.11, p<.001$, in Experiment $1 \mathrm{~B}]$. In addition, in Experiment 1B, RTs for experimental ground probes were greater than those for control ground probes [RT, $F(1,23)=4.24, p<.05]$. These findings obtained with ground probes are consistent with the prediction derived from the competitive model, in which past experience is accessed on both sides of an edge in the course of determining where the figure lies with respect to that edge.

3 . The magnitude of the impairment in shape matching for repeated grounds (control ground minus experimental ground) was larger than the benefit for repeated figures (control figure minus experimental figure) in the error data $[F(1,22)=10.52, p<.01$, and $F(1,23)=$ $6.04, p<.02$, for Experiments $1 \mathrm{~A}$ and $1 \mathrm{~B}$, respectively] and was not different in the RT data $[F(1,22)=1.05$, and $F(1,23)<1]$. This difference may arise because accuracy on the control probes may be at functional ceiling, leaving little room for increased accuracy on experimental figure probe trials.

4. Control probes facing in the direction opposite to that of the figure in the prime (i.e., control ground probes) were responded to more rapidly and more accurately
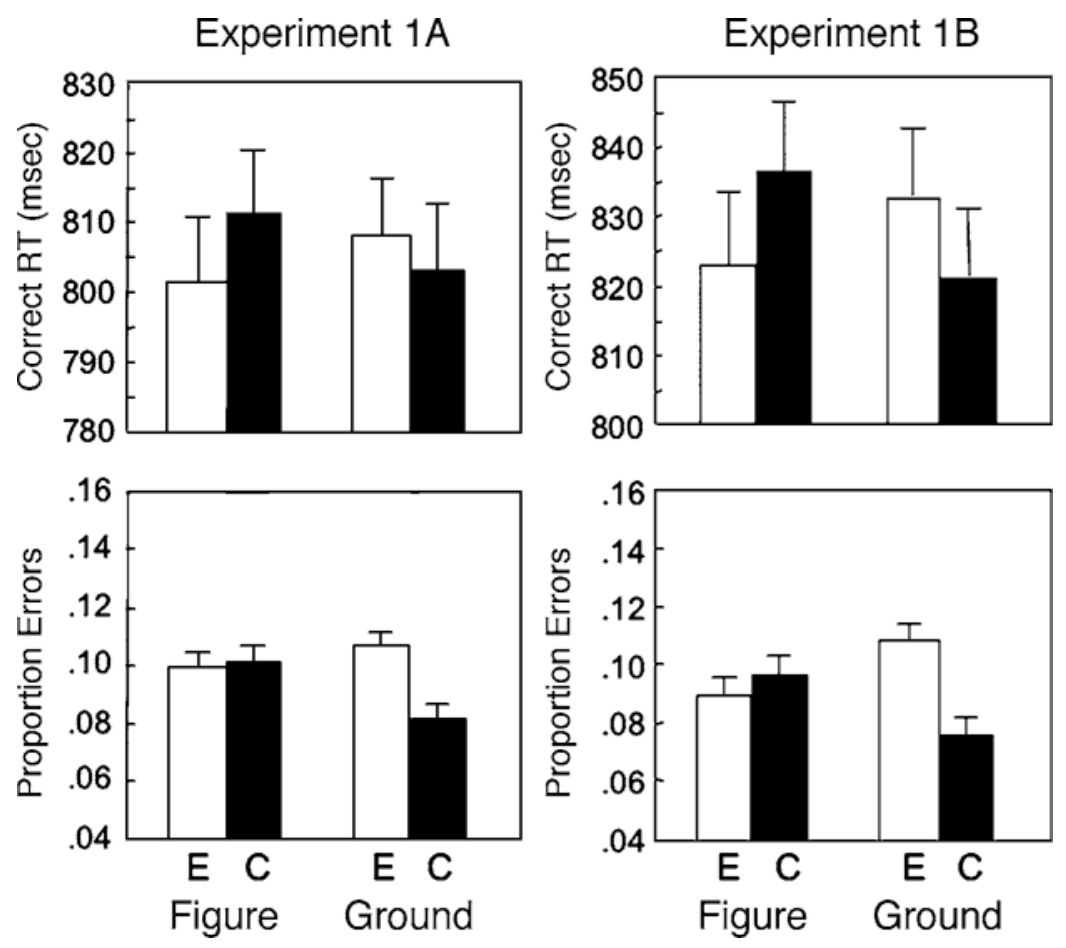

Figure 4. The results of Experiments 1A (left panel) and 1B (right panel): Mean correct response times (RTs, top panel) and proportions of errors (bottom panel) as a function of probe type (figure vs. ground) and condition. E, experimental (white bars); C, control (black bars). 
than control probes facing in the same direction as the figure in the prime (i.e., control figure probes). [For RTs, $F(1,22)=3.47, p<.07$, and $F(1,23)=6.88, p<.02$, in Experiments $1 \mathrm{~A}$ and $1 \mathrm{~B}$, respectively. For accuracy, $F(1,22)=17.95, p<.001$, and $F(1,23)=8.09, p<.01$, in Experiments $1 \mathrm{~A}$ and $1 \mathrm{~B}$, respectively.] We will defer further discussion of this unexpected finding until later in the article.

In addition to these results of primary theoretical interest, the analyses revealed main effects that are not our concern here and a few interactions of other factors with the theoretically critical interaction between probe condition and probe type. Importantly, none of these interactions tempered the main conclusions, although they are presented here for completeness.

For RTs, a probe condition $\times$ probe type $\times$ side interaction was obtained for the ground probes in Experiment $1 \mathrm{~A}[F(1,22)=4.45, p<.05]$ and for both the figure and the ground probes in Experiment $1 \mathrm{~B}[F(1,23)=$ $31.33, p<.001]$. This indicated that the difference between the experimental and the control RTs shown in Figure 4 was larger for prime figures lying on the right, rather than the left, side of fixation. This raises the possibility that edge complexes might be processed more effectively by the left than by the right hemisphere.

The remaining higher order interactions involving probe condition and probe type were found in one experiment, but not in the other. In the error data in Experiment $1 \mathrm{~A}$, there was a probe condition $\times$ probe type $\times$ color interaction $[F(1,22)=16.97, p<.001]$ and a probe condition $\times$ probe type $\times$ color $\times$ side interaction $[F(1,22)=29.27, p<.001]$, revealing larger experimental than control differences for white than for black ground probes, especially following primes on the right side. In the error data in Experiment $1 \mathrm{~B}$, a probe condition $\times$ probe type $\times$ response interaction was significant $[F(1,23)=6.15, p<.03]$, indicating that for ground probes, the magnitude of the experimental versus the control differences was quite similar for same and different responses, whereas for figure probes, the difference was larger for different responses than for same responses.

\section{Discussion}

The results of Experiments $1 \mathrm{~A}$ and $1 \mathrm{~B}$ are consistent with the hypothesis that past experience, as embodied in edge complexes, is accessed on both sides of the edge of a probe display. For experimental ground probes, the edge complex favored assigning the figure on the same side of the edge in the probe as in the prime, whereas the classic configural cues and task set favored assigning the figure on the opposite side of the edge. The edge complex thus provided cross-edge competition in experimental ground probes. This competition was reflected in longer RTs and lower accuracy, as compared with control ground probes with novel stepped edges.

In Experiment $1 \mathrm{~A}$, the unmasked prime display was exposed for only $180 \mathrm{msec}$, and the observers did not have to make an overt response to it. Nevertheless, a memory trace of where the figure lay with respect to the stepped edge was established. The present results can be joined with those of Treisman and DeSchepper (1996, Experiment 6) to demonstrate that a single exposure to a novel shape is sufficient to create an implicit memory capable of affecting figure assignment processes in the future. Treisman and DeSchepper's results were mired in controversy for some time, because they were originally interpreted within a negative-priming framework and other investigators failed to find negative priming after a single exposure to a novel shape (Grison \& Strayer, 2002; Strayer \& Grison, 1999). However, the present experiments, in concert with those reported by Peterson and Lampignano (2003), provide strong evidence that an edge complex can be established following a single prior exposure. Thus, these results indicate that certain aspects of the shape memory system are remarkably plastic. The results of Experiment $1 \mathrm{~B}$ can be added to those of Experiment $1 \mathrm{~A}$ to demonstrate that an edge complex established in a 128-msec masked exposure of a figure, to which no response was required, is still robust enough to survive masking noise.

The results of Experiments $1 \mathrm{~A}$ and $1 \mathrm{~B}$ also show that the left hemisphere may be specialized for storing edge complexes: Both positive effects for experimental figure probes and negative effects for experimental ground probes were greater following prime displays with figures on the right side, rather than on the left side. This finding was unexpected and will be investigated further in research whose aim will be to identify the neural mechanisms underlying these effects.

We also observed, unexpectedly, that control probes facing in the direction opposite to that of the figure in the prime (control ground probes) were responded to more rapidly and more accurately than control probes facing in the same direction as the figure in the prime (control figure probes). Given that this effect occurs for novel control probes, it is unlikely that it indexes differential cross-edge competition for probes facing in opposite directions. Instead, this effect may be an attentional phenomenon. Control ground probes that face in the direction opposite to that of the prime figure also lie on the opposite side of fixation. Perhaps they are more likely to be considered new objects than are control figure probes lying on the same side of fixation as the prime figure. New objects are likely to draw attention (Egeth \& Yantis, 1997). Faster responses in the control condition may reflect the differential allocation of attention to probes occurring on the same versus the opposite side of fixation as the prime figure.

\section{EXPERIMENT 2}

In Experiment 1, as in Driver and Baylis (1996), figure probes faced in the same direction as the figure in the prime, whereas ground probes faced in the opposite direction. In Experiment 2, we investigated whether the same pattern of results could be obtained when figure 
and ground probes faced in the direction opposite to their corresponding portion of the prime. Driver and Baylis did not address this question. Baylis and Cale (2001) did, but they tested only figure probes and found that responses were faster for experimental than for control figure probes, regardless of facing direction. Here, we test whether or not our effects are invariant over mirror reflections of the edge.

As can be seen in Figure 5, a reversal of facing direction is not the same as a reversal of figure and ground assignment; the former preserves shape, whereas the latter does not. Hence, experimental probes can be labeled as figure or ground probes regardless of whether they face in the same or the opposite direction as the figure in the prime (Figures 5B and 5C). All that is required for an experimental figure probe is that enclosure, small area, task set, and past experience all cue that the figure be assigned on the same side with respect to the protrusions and intrusions that make up the configuration of steps in the repeated edge as it was in the past. All that is required for an experimental ground probe is that these cues compete, with past experience favoring assigning the figure to the same side, with respect to the configuration of steps in the repeated edge, and enclosure, small area, and task set favoring assigning the figure to the opposite side.

We coded both the experimental and the control probes as to whether they faced in the same or the opposite direction as the figure in the prime (see Figure 5). Thus, in this experiment, facing direction (same as or different from that of the prime figure) was examined separately from probe type (experimental figure, experimental ground, and control). ${ }^{12}$ If we replicate the facing direction effects obtained with control probes in Experiment 1, the participants will respond more quickly to probes facing in the opposite direction, rather than in the same direction, as the figure in the prime. If the edge complex is coded in a reference frame that is reflectionally invariant, the past experience cue will be evident regardless of the facing direction of the probes. Alternatively, if following a single experience, the edge complex is coded in viewercentered coordinates, the priming effects we observed in the first two experiments will not be found when facing direction is reversed.

\section{Method}

Participants. The participants were 27 undergraduate students at the University of Arizona, who took part in order to earn class credit. The data from 5 participants were excluded because their error rates exceeded $15 \%$. Of the 22 participants whose data were analyzed, 12 were female, and 16 were right-handed.

Stimuli and Materials. The stimuli were those used in Experiment 1 , with the following changes. The facing direction of half of the experimental figure probes was mirror reversed; these probes now faced in the direction opposite to that of the figure in their paired prime. These were opposite-facing-direction figure probes. The unchanged experimental figure probes were same-facingdirection figure probes. The facing direction of half of the experimental ground probes was reversed so that they faced in the same direction as the figure in their paired prime (i.e., in the direction opposite to the ground probes used in Experiment 1). These were labeled same-facing-direction ground probes, because in Experi-

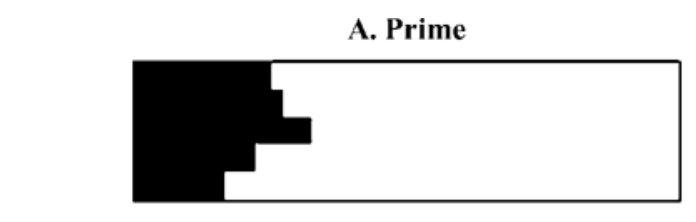

Facing Direction Relative to Prime Figure Same Opposite

B. Experimental Figure Probes

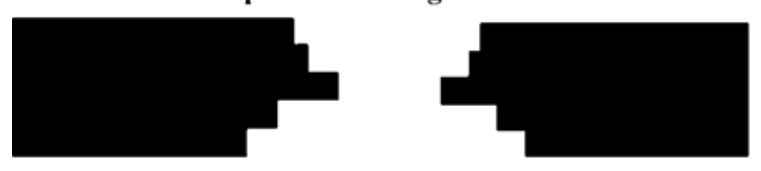

C. Experimental Ground Probes
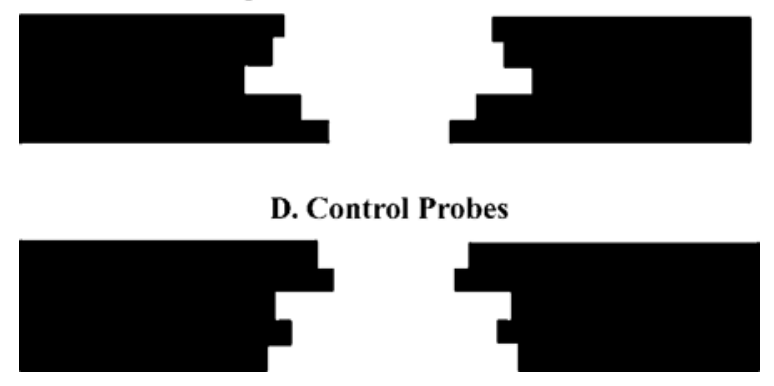

Figure 5. An illustration of how facing direction and probe type were tested in Experiment 2. Facing direction was defined with respect to the figure in the prime. For a prime figure with a stepped edge on its right side, as in panel A, probes with stepped edges on the right side face in the same direction, and probes with stepped edges on the left side face in the opposite direction. The stepped edge from the prime was repeated in experimental probes (B and C); the stepped edge in control probes (D) was novel. In each of the panels B-D, probes of the same shape are shown facing in both directions, to illustrate that the facing direction manipulation does not change shape; however, probes of the same shape were never repeated in the experiment, so as to preserve their novelty. The small, enclosed shapes in panels B-D differ from each other. The shapes in panel $D$ differ from the others because their stepped edges are different. The shapes in panels $B$ and $C$ are different even though they share the same stepped edge; they differ because the shaped entity (the figure) is assigned on opposite sides, relative to the protrusions and intrusions that make up the configuration of stepped parts along the edge. That is, the parts of the shapes in panels $B$ and $C$ have opposite polarity: Where there is a protrusion in $B$, there is an intrusion in $C$, and vice versa. Note that in panel $B$, the same-facing-direction figure probe is equivalent to an experimental figure probe in Experiment 1; the opposite-facing-direction figure probe was not tested in those experiments. In panel $\mathrm{C}$, the opposite-facingdirection ground probe is equivalent to an experimental ground probe in Experiment 1; the same-facing-direction ground probe was not tested in those experiments. In panel $D$, control probes are classified simply as facing in the same or the opposite direction as the figure in the prime; in Experiment 1, same-facing-direction control probes would have been labeled control figure probes, and opposite-facing-direction control probes would have been labeled control ground probes.

ment 2 , facing direction is labeled with respect to the figure in the prime, not with respect to the facing direction used in the previous experiments. The unchanged experimental ground probes were labeled opposite-facing-direction ground probes, because they faced in the direction opposite to that of the figure in their paired prime. 
The opposite-facing-direction ground probes in Experiment 2 faced in the same direction as the ground probes used in Experiment 1.

Half of the control ground probes used in Experiment 1 were reversed to form half of the set of same-direction control probes; half of the control figure probes from Experiment 1 made up the other half of the same-direction control probe set. Similarly, half of the control figure probes used in Experiment 1 were reversed to form half of the set of opposite-direction control probes; half of the control ground probes from Experiment 1 made up the other half of the opposite-direction control probe set. If the speeded responses to control probes facing in the opposite direction from that of the figure in the prime that were observed in Experiment 1 are replicated here, we can be more confident that this effect was not due to differences in the shape of the control probes.

Procedure. The procedure was the same as that in Experiment 1B, with the time-out duration set to $2,000 \mathrm{msec}$.

\section{Results}

Before the RT data were analyzed, trials on which errors were made or the time-out interval was exceeded were excluded. Recursive removal of any RTs more than two standard deviations from each participant's mean for each condition eliminated $8.7 \%$ of the responses.

Mean correct RTs and mean proportions of errors are shown in Figure 6. An ANOVA was used to examine the following factors: facing direction with respect to the figure in the prime (same or opposite), probe type (experimental figure, experimental ground, or control), probe color (black or white), response (same or different), and side of figure in the prime display (left or right). For clarity, arrows at the bottom of Figure 6 indicate which comparisons are the same as those in Experiment 1 (old comparisons) and which comparisons involve mirror reflected shapes (new comparisons). The main effect of probe type was significant for both RT $[F(2,42)=8.36$, $\left.M S_{\mathrm{e}}=2,953.892, p<.001\right]$ and errors $[F(2,42)=12.01$, $\left.M S_{\mathrm{e}}=0.003, p<.001\right]$. This reflected generally faster and more accurate responding to experimental figure probes than to control probes [RT, $F(1,42)=4.62, p<$ .04 ; errors, $F(1,42)=15.74, p<.001]$ and generally slower and less accurate responses to experimental ground probes than to control probes [RT, $F(1,42)=3.76, p<$ .06 ; errors, $F(1,42)=20.04, p<.001]$. Thus, the effects observed in Experiment 1 were robust over a change in facing direction.

As in the previous two experiments, the magnitude of the impairment in shape matching for experimental ground probes (control probes minus experimental ground probes) was larger than the benefit for repeated figures (control figure minus experimental figure) in the error data $[F(1,42)=8.28, p<.01]$ and was not different in the RT data $[F(1,42)<1]$. (Again, this may have been due to a functional floor effect in errors made to repeated figure probes.)

The main effect of facing direction was significant for RT $\left[F(1,42)=32.84, M S_{\mathrm{e}}=2,612.461, p<.001\right]$, replicating the facing direction effect observed in responses to control probes in Experiment 1. This main effect reflected generally faster responses to probes facing in the opposite, rather than the same, direction as the figure in the prime display. The facing direction effect was ob-
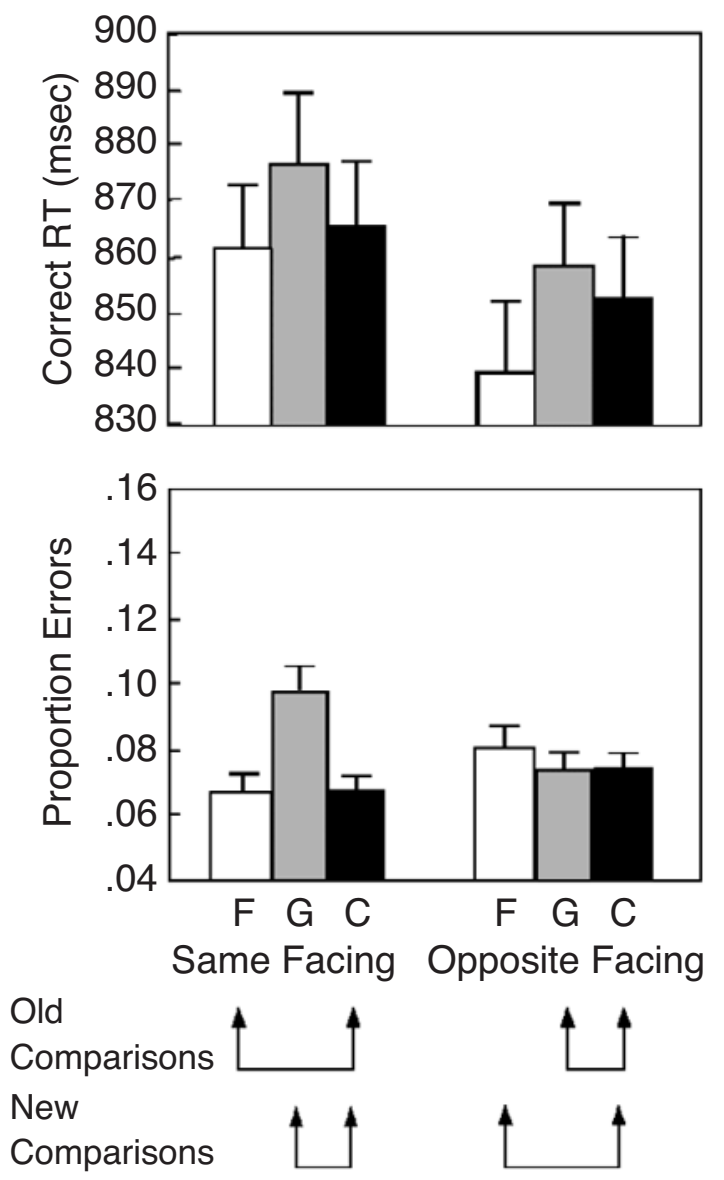

Figure 6. The results of Experiment 2. Mean correct response times (RTs, top panel) and proportions of errors (bottom panel) as a function of facing direction (same vs. opposite direction as that of the figure in the prime) and probe type ( $F$, experimental figure, white bars; G, experimental ground, gray bars; and $\mathbf{C}$, control, black bars). The comparisons labeled old are the same as those in Experiments $1 \mathrm{~A}$ and $1 \mathrm{~B}$, which involved same-facing figure and control probes and opposite-facing ground and control probes. The comparisons labeled new are those involving a mirror reversal between prime and probe displays.

tained for all probe types in RT. The probe type $\times$ facing direction interaction was not significant in the RT data $(F<1)$. In the error data, facing direction was not significant as a main effect $(F<1)$, but it interacted with probe type $\left[F(1,42)=17.20, M S_{\mathrm{e}}=0.003, p<.001\right]$. This interaction was due entirely to less accurate responding to ground probes than to control probes when they faced in the same direction as the figure in the prime display [i.e., in the opposite direction from the ground probes used in Experiment 1; Fisher LSD, $F(1,42)=$ $8.09, p<.01]$.

In the ANOVAs for both RTs and errors, the highest order probe type $\times$ facing direction $\times$ probe color $\times$ side $\times$ response interaction was significant $[\mathrm{RT}, F(2,42)=$ $5.77, p<.01$; errors, $F(2,42)=8.12, p<.001$ ], in addition to several other component interactions. As in the previous two experiments, these interactions seemed to 
arise from synergistic effects among the factors of side of prime (larger control minus experimental differences for right- than for left-side primes), probe color (larger control minus experimental differences for white than for black probes), and response (larger control minus experimental differences for different than for same trials) that occurred over and above the primary effects of interest.

\section{Discussion}

Once again, we found that the observers responded more quickly to control probes that faced in the direction opposite to that of the figure in the prime than to control probes that faced in the same direction. In addition, we found that, regardless of facing direction, the observers responded more quickly and more accurately to experimental figure probes than to control probes and more slowly and less accurately to experimental ground probes than to control probes. The results of Experiment 2 show that the effects observed in Experiment 1 do not depend on facing direction. These results also show that the edge complex is not coded in viewer-centered coordinates. Instead, the data show that the change in figure assignment was responsible for the different RTs and error rates obtained for experimental figure and experimental ground probes in Experiment 1, not the change in facing direction. Had the edge complex been coded in viewer-centered coordinates, the effects would not have survived the change in facing direction. Instead, the edge complex seems to specify where the figure was previously perceived with respect to the configuration of steps along a particular edge. That configuration remained the same despite a change in facing direction.

\section{GENERAL DISCUSSION}

In this study, we examined memory for a figure that was seen briefly and only once in a prime display. In the present experiments, there was no formal need to evaluate either side of the prime display as belonging to a figure or a ground, no need to respond to the figure, and no need to remember the edge itself. Nevertheless, our results indicate that the observers saw the small bright region in the prime as the figure and the larger, dimmer region as the ground and that their memory of these assignments influenced subsequent responses. These results support Driver and Baylis's (1996) claim that figure assignment occurs even when it is not demanded by the observer's task.

These experiments were designed to investigate whether the pattern of slower responses to ground probes than to figure probes obtained by Driver and Baylis (1996) could have indicated that the ground side of an edge is processed for shape attributes prior to figure assignment, contrary to their conclusion that it is not. We argued that their results were equivocal until the RTs to figure and ground probes with repeated edges could be compared with RTs to control figure and ground probes with novel edges. Like Driver and Baylis (1996), we repeated the prime edge under two conditions: one in which it was shown as bounding a shape lying on the same side as in the prime (experimental figure probes), and one in which it was shown as bounding a shape lying on the side that has been seen as the ground in the prime (experimental ground probes). Unlike Driver and Baylis, we asked observers to judge whether two shapes presented simultaneously on the probe trials were the same as or different from each other, and we included both figure and ground control probes with completely novel edges.

We found that the observers' RTs were shorter when the repeated edge was presented as bounding a shape lying on the side that was specified to be figure in the prime, as compared with matched control trials. These are standard priming effects. However, unlike Baylis and Cale (2001), and contrary to the conclusions of Driver and Baylis (1996), we found that RTs were longer, as compared with matched control trials, when the repeated edge was presented as bounding a shape lying on the side that had previously been seen as ground in the prime.

We interpret the results in the present study as strong evidence that edge complexes are accessed on both sides of the probe edge in the process of figure assignment. It has previously been assumed that figure cues that do not require past experience (e.g., the classic configural cues and the part saliency cue more recently identified by Hoffman \& Singh, 1997) are assessed on both sides of an edge prior to figure assignment. The present results show that the same is true for the past experience cue, as Peterson has claimed (see, e.g., Peterson \& Gibson, 1994; Peterson \& Kim, 2001). Furthermore, the negative effects obtained with experimental ground probes are entirely consistent with a competitive model, if one assumes that the outcome of the competition that occurs across the prime edge is stored in a memory trace-an edge complex - and that the edge complex enters into the crossedge competition the next time the edge is encountered, voting for assigning the figure to the same side of the edge as it was on in the past.

\section{Will All Tasks Show Evidence of Cross-Edge Competition?}

The longer RTs we obtained for ground probes are an indirect measure of the ambiguity of an edge. It is possible that this ambiguity can be measured only with certain tasks. If we assume that observers attempt to generate a response as quickly as they can (e.g., on the basis of the lowest level information possible), the fact that a different shape is potentially present on the opposite side of an edge will interfere with some tasks, but not with others, because, in some tasks, the determination of where the task-relevant shape lies with respect to an edge must be made before a response can be generated; in other tasks, the response may be made without this determination.

Consistent with this notion, evidence for cross-border competition (edge ambiguity) was obtained by Treisman and DeSchepper (1996), Peterson and Lampignano (2003), and the present experiments because the potentially different shape suggested on the opposite side of the edge of the ground probes could interfere with same/different responses. To avoid this response interference, the cross- 
edge competition had to be resolved before the response to the task could be generated (see Peterson \& Lampignano, 2003, for a more extended discussion).

Recall that Baylis and Cale (2001) failed to find RT differences between experimental and control ground probes. In the introduction, we mentioned some ways in which Baylis and Cale's experiment differed from previous experiments that did show RT differences between experimental and control ground probes. From the present experiments, we know that some of those factors are irrelevant. One task difference that may be relevant is that Baylis and Cale asked observers to judge whether or not horizontally elongated stimuli with one complex curvilinear edge were symmetric around a vertical axis. Since the shapes potentially present on both sides of the edge were both symmetric or both asymmetric, it was not formally necessary for the observers to resolve which side of the edge was figure, as opposed to ground, before they generated a response. There may have even been a "horse race" for the symmetry judgment based on both sides of the edge.

Investigations of which tasks reveal effects from past experience and which do not must be continued. Such research has already begun in other domains. For instance, Liu and Cooper (2001) have shown that the likelihood of observing priming is affected by task requirements.

Our experiments measured the influence of past experience implicitly by showing that response latencies are longer and accuracy is lower when past experience competes with classic configural cues and task set, as would be predicted by a competitive model that assumes that figure assignment takes time and that the amount of time required increases as cross-edge competition increases. We note that because edge complexes do not necessarily determine the perceived figure when competing cues are present, explicit tasks may not be good measures of their existence under such conditions.

\section{What and Where Are Edge Complexes?}

The edge complexes that are proposed as the basis for the implicit priming observed in this study are probably coded in posterior ventral cortical regions that are activated early in the feedforward sweep of processing (Peterson, 2003). This conjecture is based on research showing that past experience affects figure assignment only if it is available early in the course of processing (for a review, see Peterson \& Skow-Grant, 2003). We note that edge complexes are not assumed to represent shapes or objects holistically. Rather, following multiple exposures to an object under different viewing conditions, many overlapping edge complexes are likely to be established for a single object, each representing different subconfigurations along the bounding edge of the object (Peterson, 2003). Experiment 2 suggests further that the edge complexes are invariant over mirror reflections. Peterson (2003) has also proposed that individual features or parts are represented redundantly in more than one edge complex. The proposed overlapping nature of the edge complexes suggests a means for coding the location of the shaped entity with respect to an edge. Activation of one edge complex might activate other edge complexes for that shape. The ensemble of activated edge complexes may then specify where the shaped entity lies, relative to the present edge.

Our proposals regarding overlapping edge complexes are very similar to proposals developed in computational and physiological research. Edelman and Intrator (2000, 2003) proposed that a "chorus of fragments" might represent objects at intermediate levels. Ullman, VidalNaquet, and Sali (2002) showed that a model using overlapping intermediate complexity fragments extracted from past experience can successfully accomplish both object classification and scene segmentation. Perrett and Oram (1998) made a similar proposal for intermediatelevel face representations.

Physiological evidence supporting the idea of intermediate complexity shape representations has been reported by Tanaka (1996) in monkeys. Recently, using fMRI with humans, Grill-Spector et al. (1998; Lerner, Hendler, Ben-Bashat, Harel, \& Malach, 2001) found that intermediate visual areas, such as V3, Vp, and the lateral occipital complex (LOC), responded equivalently to pictures of whole objects and the same objects broken into 16 subregions, but less vigorously to pictures of objects broken into smaller pieces. Thus, there is some evidence that configured fragments of objects are represented at intermediate levels in the human brain. Research by others suggests that the representations mediating past experience effects on figure assignment are not likely to lie in more anterior ventral regions. Baylis and Driver (2001) showed that single neurons in the anterior-inferior temporal cortex show figure-priming effects, but not groundpriming effects. Similarly, using the slower population response recorded by fMRI, Kourtzi and Kanwisher (2001) found that the LOC treats shapes such as our experimental ground and control ground probes similarly. We conjecture that the cells recorded by Baylis and Driver and the brain response indexed by Kourtzi and Kanwisher reflect the perceived shape of the figure, not the cross-edge competition. We expect that the competition would be measurable in other brain regions or when different techniques were used (e.g., we are currently using ERP techniques with better temporal resolution than fMRI has). In addition, we are currently exploring the interesting possibility raised by the present results that edge complexes are processed more efficiently in the left hemisphere than in the right hemisphere.

\section{Competitive Models of Figure-Ground Segregation}

Peterson et al.'s (2000) model is similar to competitive models of figure-ground segregation proposed by others (Kienker, Sejnowski, Hinton, \& Schumacher, 1986; Sejnowski \& Hinton, 1987; Vecera \& O’Reilly, 1998, 2000). The extant competitive models differ in whether or not they explicitly allow a role for past experience; the only ones that do are those proposed by Peterson et al. and by Vecera and O'Reilly. Despite the differences among them, 
all of the extant competitive models assume that both sides of an edge are processed prior to figure assignment. The present experiments add to the small body of behavioral research confirming predictions of competitive models in general and, in particular, those that include past experience among the factors contributing to figure-ground segregation (Peterson \& Kim, 2001; Peterson \& Lampignano, 2003). Research designed to test other predictions of these competitive models is under way.

\section{REFERENCES}

BAYlis, G. C., \& CALE, E. (2001). The figure has a shape, but the ground does not: Evidence from a priming paradigm. Journal of Experimental Psychology: Human Perception \& Performance, 27, 633-643.

BAYLIS, G. C., \& DRIVER, J. (1995). One-sided edge assignment in vision: 1. Figure-ground segmentation and attention to objects. Current $D i-$ rections in Psychological Science, 4, 140-146.

BAYLIS, G. C., \& DrIVER, J. (2001). Shape coding in IT cells generalizes over contrast and mirror reversal but not figure-ground reversal. Nature Neuroscience, 4, 937-942.

Beller, H. K. (1971). Priming: Effects of advance information on matching. Journal of Experimental Psychology, 87, 176-182.

Belongie, S., Malik, J., \& Puzicha, J. (2002). Shape matching and object recognition using shape contexts. IEEE Transactions on Pattern Analysis \& Machine Intelligence, 24, 509-521.

BIEDERMAN, I. (1987). Recognition-by-components: A theory of human image understanding. Psychological Review, 94, 115-147.

Dosher, B. A., Sperling, G., \& Wurst, S. A. (1986). Tradeoffs between stereopsis and proximity luminance covariance as determinants of perceived 3D structure. Vision Research, 26, 973-990.

DRIVER, J., \& BAYLIS, G. C. (1995). One-sided edge assignment in vision: 2. Part decomposition, shape description, and attention to objects. Current Directions in Psychological Science, 4, 201-206.

Driver, J., \& BAYLIS, G. C. (1996). Edge-assignment and figure-ground segmentation in short-term visual matching. Cognitive Psychology, 31, 248-306.

Edelman, S., \& Intrator, N. (2000). (Coarse coding of shape fragments $)+($ retinotopy $)=$ representation of structure. Spatial Vision, 13, 255-264.

EDELMAN, S., \& INTRATOR, N. (2003) Towards structural systematicity in distributed, statically bound visual representations. Cognitive Science, 27, 73-109.

Egeth, H. E., \& Yantis, S. (1997). Visual attention: Control, representation, and time course. Annual Review of Psychology, 48, 269-297.

Forster, K. I., \& Forster, J. C. (2003). DMDX: A Windows display program with millisecond accuracy. Behavior Research Methods, Instruments, \& Computers, 35, 116-124.

Grill-Spector, K., Kushnir, T., Hendler, T., Edelman, S., ITZCHAK, Y., \& MALACH, R. (1998). A sequence of object-processing stages revealed by fMRI in the human occipital lobe. Human Brain Mapping, 6, 316-328.

Grison, S., \& StraYer, D. L. (2002). Negative priming and perceptual fluency: More than what meets the eye. Perception \& Psychophysics, 63, 1063-1071.

HochberG, J. (1971). Perception I: Color and shape. In J. W. Kling \& L. A. Riggs (Eds.), Woodworth and Schlossberg's experimental psychology (3rd ed., pp. 395-474). New York: Holt, Rinehart, \& Winston.

Hoffman, D. D., \& Singh, M. (1997). Salience of visual parts. Cognition, 63, 29-78.

JACOBY, L. L. (1983). Perceptual enhancement: Persistent effects of an experience. Journal of Experimental Psychology: Learning, Memory, \& Cognition, 9, 21-38.

KANIZSA, G., \& GERBINO, W. (1976). Convexity and symmetry in figureground organization. In M. Henle (Ed.), Vision and artifact (pp. 2532). New York: Springer-Verlag.

KienKer, P. K., Sejnowski, T. J., Hinton, G. E., \& Schumacher, L. E. (1986). Separating figure from ground with a parallel network. Perception, 15, 197-216.
Kourtzi, Z., \& Kanwisher, N. (2001). Representation of perceived object shape by the human lateral occipital complex. Science, 293, 1506-1509.

Lerner, Y., Hendler, T., Ben-Bashat, D., Harel, M., \& Malach, R. (2001). A hierarchical axis of object processing stages in the human visual cortex. Cerebral Cortex, 11, 287-297.

LiU, T., \& CoOper, L. A. (2001). The influence of task requirements on priming in object decision and matching. Memory \& Cognition, 29, 874-882.

Palmer, S. E. (1999). Vision science: Photons to phenomenology. Cambridge, MA: MIT Press.

Perrett, D. I., \& Oram, M. W. (1998). Visual recognition based on temporal cortex cells: Viewer-centred processing of pattern configuration. Zeitschrift für Naturforschung, 53, 518-541.

Peterson, M. A. (1999). Organization, segregation and object recognition. Intellectica, 28, 37-51.

Peterson, M. A. (2000). Object perception. In E. B. Goldstein (Ed.), Blackwell handbook of perception (pp. 168-203). Malden, MA: Blackwell.

Peterson, M. A. (2003). Overlapping partial configurations in object memory: An alternative solution to classic problems in perception and recognition. In M. A. Peterson \& G. Rhodes (Eds.), Perception of faces, objects, and scenes: Analytic and holistic processes (pp. 269294). New York: Oxford University Press.

Peterson, M. A., De Gelder, B., Rapcsak, S. Z., Gerhardstein, P. C., \& BACHOUd-LÉvI, A.-C. (2000). Object memory effects on figure assignment: Conscious object recognition is not necessary or sufficient. Vision Research, 40, 1549-1567.

Peterson, M. A., \& Gibson, B. S. (1994). Must shape recognition follow figure-ground organization? An assumption in peril. Psychological Science, 5, 253-259.

Peterson, M. A., \& Kim, J. H. (2001). On what is bound in figures and grounds. Visual Cognition, 8, 329-348.

Peterson, M. A., \& Lampignano, D. W. (2003). Implicit memory for novel figure-ground displays includes a history of cross-border competition. Journal of Experimental Psychology: Human Perception \& Performance, 29, 808-822.

Peterson, M. A., \& Skow-Grant, E. (2003). Memory and learning in figure-ground perception. In D. Irwin \& B. Ross (Eds.), Psychology of learning and motivation: Cognitive vision (Vol. 42, pp. 1-34). San Diego: Academic Press.

Pomerantz, J. R., \& Kubovy, M. (1986). Theoretical approaches to perceptual organization: Simplicity and likelihood principles. In K. R. Boff, L. Kaufman, \& J. Thomas (Eds.), Handbook of perception and human performance: Vol. 2. Cognitive processes and performance (pp. 1-46). Oxford: Wiley.

Rosch, E. (1975). Cognitive representations of semantic categories. Journal of Experimental Psychology: General, 104, 192-233.

Rubin, E. (1958). Figure and ground. In D. C. Beardslee \& M. Wertheimer (Eds. and Trans.), Readings in perception (pp. 35-101). Princeton, NJ: Van Nostrand. (Original work published 1915)

Scarborough, D. L., Cortese, C., \& Scarborough, H. S. (1977). Frequency and repetition effects in lexical memory. Journal of Experimental Psychology: Human Perception \& Performance, 3, 1-17.

Sejnowski, T. J., \& Hinton, G. E. (1987). Separating figure from ground with a Boltzmann machine. In M. Arbib \& A. Hanson (Eds.), Vision, brain, and cooperative computation (pp. 703-724). Cambridge, MA: MIT Press.

SeKuler, A. B., \& Palmer, S. E. (1992). Perception of partly occluded objects: A microgenetic analysis. Journal of Experimental Psychology: General, 121, 95-111.

StraYer, D. L., \& Grison, S. (1999). Negative identity priming is contingent on stimulus repetition. Journal of Experimental Psychology: Human Perception \& Performance, 25, 24-38.

TANAKA, K. (1996). Inferotemporal cortex and object vision. Annual Review of Neuroscience, 19, 109-139.

Treisman, A., \& DeSchepper, B. (1996). Object tokens, attention, and visual memory. In T. Inui \& J. L. McClelland (Eds.), Attention and performance XVI: Information integration in perception and communication (pp. 15-46). Cambridge, MA: MIT Press.

Ullman, S., Vidal-Naquet, M., \& Sali, E. (2002). Visual features of 
intermediate complexity and their use in classification. Nature Neuroscience, $\mathbf{5}, 682-687$.

VeCERA, S. P., \& O'ReILLY, R. C. (1998). Figure-ground organization and object recognition processes: An interactive account. Journal of Experimental Psychology: Human Perception \& Performance, 24, 441-462.

VeCERA, S. P., \& O'ReILLY, R. C. (2000). Graded effects in hierarchical figure-ground organization: Reply to Peterson (1999). Journal of Experimental Psychology: Human Perception \& Performance, 26, 12211231.

\section{NOTES}

1. We are aware that it is possible for a region to be figure along some portions of its bounding edge and ground along other portions. An example is a partially occluded object, such as a person sitting behind, and partially occluded by, a desk.

2. Although brightness per se has not been shown to be a configural cue, it has been shown to be a depth cue: Bright regions are likely to be seen as near regions (Dosher, Sperling, \& Wurst, 1986), as are figures.

3. In Driver and Baylis (1996), left and right relative to the observer and side relative to the particular configuration of protrusions and intrusions along the stepped edge were confounded, but this need not be the case (see Experiment 3).

4. Treisman and DeSchepper (1996) did propose that shape descriptions are formed for the shapes potentially present on both sides of an edge prior to figure assignment, although Peterson and colleagues never did. Peterson and Lampignano (2003) presented a different interpretation of the results that led Treisman and DeSchepper to make this claim.

5. It could as well code the outcome of the competition in processing weights.

6. In Treisman and DeSchepper's (1996) study, the black standard shape on the prime trial was shown against a white ground, approxi- mately equal in area to the black figural region, and both were shown on a larger gray background. The white experimental ground probes had the same size and overall shape as the white regions putatively seen as grounds in the prime trials. In Peterson and Lampignano's (2003) study, the white ground was not present on the prime trials.

7. This is a standard interpretation of results obtained using a negativepriming paradigm and is very similar to the interpretation Treisman and DeSchepper (1996) applied to their own results.

8. Some of these participants were excluded because our computer program was set to reject RTs longer than $1,500 \mathrm{msec}$ and to count these rejected trials as errors. Between-block feedback encouraged the participants with high errors (the error count included trials rejected because of long RTs) to try to be more accurate. A natural tendency when trying to be more accurate is to slow down, thereby increasing the number of trials rejected because of long RTs. In Experiments 2 and 3, we recorded responses with longer RTs; this reduced our rejection rate.

9. The number of trials and the length of the practice block were modeled on Driver and Baylis (1996).

10. A 1,500-msec time-out period was used for 10 participants in Experiment 1B. An ANOVA comparing the mean RTs for these 10 participants with those for the 14 participants run with a time out of $2,000 \mathrm{msec}$ showed no main effects or interactions.

11. Driver and Baylis (1996) excluded $10.6 \%$ of trials in an experiment in which conditions that were most similar to those in Experiment $1 \mathrm{~A}$ were used.

12. Control probes are no longer labeled as control figure and control ground probes, as in Experiment 1.

(Manuscript received January 7, 2004; revision accepted for publication September 13, 2004.) 